\title{
Creation of arteriovenous fistula for hemodialysis in the older population
}

\author{
Monika Medricka ${ }^{a}$, Jana Janeckovaa ${ }^{a}$ Julia Jarosciakova ${ }^{a}$, Petr Bachleda ${ }^{a}$
}

\begin{abstract}
Objective. To evaluate the results of arteriovenous fistula (AVF) created for haemodialysis in patients older than 65 years of age.

Subjects and Methods. A retrospective analysis of patients with AVF or arteriovenous graft (AVG) creation, who were older than 65 years of age and were operated on at the II. Surgical Clinic at the University Hospital in Olomouc from 2014 - 2018 was performed.

Results. 212 patients were evaluated and a total of 239 AVF/AVG were created. 194 AVFs (81.18\%) and 45 AVGs (18.82\%) were created. Primary failure was seen in 19 arteriovenous fistulas (9.8\%) and 2 arteriovenous grafts (4.44\%). The primary patency of AVF was $69.9 \%, 62.8 \%$ after 12 and 24 months, respectively, and in the case of AVG it was $54.7 \%$ and $32.3 \%$ after 12 and 24 months, respectively. Primarily assisted patency of AVF was $77.6 \%$ and $66.3 \%$ after 12 and 24 months, respectively, and in case of AVG it was $69.1 \%$ and $39.7 \%$ after 12 and 24 months, respectively. Secondary patency of AVF was $77.6 \%$ and $66.3 \%$ after 12 and 24 months, respectively, and for AVG it was $69.1 \%$ and $39.7 \%$ after 12 and 24 months, respectively.

Conclusion. The type of vascular access should be selected based on a thorough, protocol-based examination. In most seniors, AVF is the method of choice. The AVG is a suitable choice for patients with an exhausted venous bed, in acute need of haemodialysis, in the elderly and in females. A "customized" approach should be matter of fact for older generations.
\end{abstract}

Key words: renal disease, end stage, hemodialysis, arteriovenous fistula, arteriovenous graft, elderly

Received: January 8, 2020; Revised: March 4, 2020; Accepted: March 13, 2020; Available online: April 7, 2020

https://doi.org/10.5507/bp.2020.013

(c) 2021 The Authors; https://creativecommons.org/licenses/by/4.0/

${ }^{a}$ Department of Vascular and Transplantation Surgery, University Hospital Olomouc, Czech Republic Corresponding author: Jana Janeckova e-mail:jana.fialova@email.cz

\section{INTRODUCTION}

Hemodialysis (HD) is the most commonly used method in RRT - renal replacement therapy. Access for HD is ensured by a CVC - central venous catheter, arteriovenous fistula (AVF) or arteriovenous graft (AVG). Based on the current AVF guidelines, the method of choice is the fistula-first ${ }^{1}$. AVF is preferred because there are fewer complications. An arteriovenous graft (AVG) is indicated in patients with failed AVF, exhausted superficial venous bed or unsuitable vessels. However, the reported benefits of AVF in comparison with AVG are less obvious in older patients because the biological changes associated with age affect the results independently. Use of CVC is an easy approach to HD. However, it is associated with the highest incidents of complications and is primarily used for patients in the initial phases of $\mathrm{HD}$, when there is no time to create an $\mathrm{AV}$ approach.

The aim of this paper is to evaluate the effectiveness of establishing an arteriovenous access for HD in patients older than 65 years of age, who were operated on at the II. Surgical Clinic at the University Hospital in Olomouc in a 5-year period and compare both types of AV accesses in relation to age, diabetes and gender on patency.

\section{Methodology}

A retrospective study evaluated all patients with end stage renal disease - ESRD, who were older than 65 years of age and AV creation was performed at the II. Surgical Clinic at the University Hospital in Olomouc from January 2014 - December 2018.

Prior to creation of the vascular access to HD, we used a standardized, protocol-based examination, which also included a clinical examination of the upper extremities, an examination of the arterial and venous system using Doppler ultrasonography (DUS) and phlebography before AVG creation or if the findings were unclear. The patient's future vascular access was selected on an individual basis, according to these examinations. AVG creation was primarily indicated if the pre-surgical findings on the vascular bed or on the DUS were unsatisfactory and in patients with a longer life expectancy, upon a borderline finding of the vascular bed on the DUS. Secondly, AVG was indicated during primary failure of the AVF. In the case of AVF primary failure, the patient was re-evaluated and an AVF was created proximally in most cases. The AVF or AVG was indicated on the other extremity. The strategy of these and consequent operations was individualized based on the necessity to commence HD. The AVG configurations used were brachio-brachial, radio-cephalic and brachio-cephalic. We used a prosthesis made of ex- 
panded polytetrafluorethylene (ePTFE) made by Rapidax (VASCUTEK Ltd. A TERUMO Company) or Acuseal $\left(\right.$ Gore $\left.^{\circledR}\right)$, which are cannulated early.

All patients were hospitalized for a short time. The surgery was performed under local or regional anaesthesia. If vascular prosthesis were used, antibiotics were administered prophylactically (pre and post-surgery), patients were provided with anti-aggregation therapy by acetylsalicylic acid and anti-thrombosis sulodexide after the surgery. The vascular access was clinically examined before the patients were discharged from hospital. After being discharged, clinical check-ups included the removal of stitches 7-10 days after surgery. The patients were clinically examined using the DUS and the patency of the AV access were measured 6 weeks after the vascular access was created. Patients with the AVG then regularly came in for check-ups every 6 weeks.

\section{Definitions and statistical processing}

Primary failure of the AV access was defined as the inability to utilize the AV access for dialysis for regular dialysis treatment, because of insufficient AVF maturation or insufficient flow within 3 months of primary creation. The insufficient AVF flow was specified at $0.5 \mathrm{~L} / \mathrm{min}$.

An intervention-free access survival was defined as the interval from the time the vascular access was created to any intervention indicated to maintain the function of the vascular access, for renewing patency, or until the vascular access closed (thrombosis).

The thrombosis-free access survival was defined as the interval from the time the vascular access was created to the time it was closed (thrombosis), including interventions (surgical or endovascular) indicated to maintain function of the vascular access.

Survival of the access until abandonment was defined as the interval from the time the vascular access was created until its abandonment, including interventions (surgical or endovascular) indicated to maintain the functionality of the vascular access ${ }^{2}$.

The data was analyzed using IBM SPSS Statistics version 22 (USA). Kaplan-Meier analysis with Log-rank test and Cox regression analysis were used to evaluate the significance of the individual factors during patency. The test results were presented as a hazard ratio (HR) with $95 \%$ reliability intervals (coincidence interval - CI). The tests were performed on a 0.05 significance level. The chi-quadrate test (Fisher's accuracy test) was used to compare the number of interventions up to 3 months and up to 12 months.

Manipulation with the patients' data in the monitored group was anonymous.

\section{RESULTS}

\section{Patient Population}

A total of 480 patients with ESRD were operated on at our department during the monitored period. The monitored population comprised of $44.2 \%$ of patients older than 65 . The study included 212 patients over 65, who had 239 AV approaches for HD established from January 2014 to December 2018.

A total of 194 AVFs (81.18\%) were created, 49 of the AVF were radio-cephalic (20.5\%), 68 were brachio-cephalic (28.45\%), 24 were brachio-basilic (10.05\%) and 72 were brachio-cephalic-basilic (30.12\%). Of the 45 AVGs (18.82\%) created, 41 were brachio-brachial (91.1\%), 2 were radio-cephalic $(4.44 \%)$ and 2 were brachio-cephalic (4.44\%).

Of the 212 patients, 72 were women and 140 were men, the median age was 74 (ranging from 65-97 years of age). 131 patients (62\%) had diabetes mellitus (DM). In 101 patients (42.2\%), AV access was via a CVC until the HD access was created. There were 26 deaths and 8 transplantations during the monitoring period.

\section{AVF}

Early failure occurred in 5 radio-cephalic AVFs, 5 radio- cephalic AVFs, 3 brachio- cephalic AVFs, 1 brachiobasilic AVF and 5 brachio-cephalic-basilic AVFs within 3 months. In total, failure occurred in 19 AVFs (9.8\%). 6 AVFs had extended maturation (all were radio-cephalic AVFs). After the AVF failed, 36 patients had CVC due to an overall poor condition, where establishing another $\mathrm{AV}$ access was not indicated. HD treatment was not even commenced in patients with AVF because the progression of kidney problems stopped.

\section{AVG}

6 AVGs were primarily indicated, these patients did not have a CVC at the time of creation. Primary failure occurred in 2 AVGs (4.44\%). 39 AVGs (16.3\%) were indicated after failure of the previous AVF, HD occurred via CVC for all of them. More than one AVG was established in 6 patients. The Acuseal prosthesis was used 10 times and the Rapidax prosthesis was used 35 times.

\section{Patency of the AV access}

Primary patency of all AV accesses created was $69.2 \%$ and 56.6\% after 12 and 24 months, respectively. Primary patency divided by AVF and AVG after 12 and 24 months is specified in Table 1. Primary assisted patency of all AV accesses created was $71.6 \%$ and $62.74 \%$ after 12 and 24 months, respectively. Primary assisted patency divided by AVF and AVG after 12 and 24 months is specified in Table 2. Secondary patency of all AV accesses created was $75.9 \%$ and $61.4 \%$ after 12 and 24 months, respectively. Secondary patency divided by individual type of AV access is specified in Table 3 .

When comparing secondary patency, there was a significantly longer period of secondary patency with AVF (mean 49 months, median 45 months) than with AVG (mean 25 months, median 20 months), Log-rank test $P=0.023$.

The effect of DM on the secondary patency period was not confirmed in neither the AVF (Log-rank test $P=0.790)$. OR=1.072 (95\% CI: $0.639-1.800)$ nor in the AVG (Log-rank test $P=0.987$ ) group.

There was a significantly shorter secondary patency of AVF in women (mean 31 months) than in men (mean 
55.6 months), Log-rank test $P=0.029$. The effect of gender on the period of secondary patency of AVG was not confirmed (Log-rank test $P=0.711$ ).

A significantly shorter period of secondary patency of AVF was confirmed in patients older than 74 (mean 35 months) than patients younger than 74 (mean 62 months), Log-rank test $P=0.001$. The effect of age on the period of secondary patency of AVG was not confirmed (Log-rank test $P=0.527$ ).

When evaluating early surgical intervention or endovascular intervention, a significantly higher number was observed (primary assisted patency) for the AVG up to 3 months (23\%) in comparison with the AVF (4.8\%), Fisher's accuracy test $P=0.047$; also up to 12 months (69\%) in comparison with the $\operatorname{AVF}(34 \%)$, chi-quadrant test $P=0.005$.

\section{DISCUSSION}

The fistula-first approach is based on retrospective analyses, which were conducted over 20 years ago, when the elderly made up less than $15 \%$ of the ESRD population $^{3,4}$. The age of patients with ESRD is increasing worldwide and the numbers are increasing every year. According to European registers, almost half of the patients are older than 65 (ref. $\left.{ }^{5}\right)$. Our results also confirm this data because almost half of our patients with ESRD were older than 65 . The rigid fistula-first is slowly becoming obsolete in this older population and the newer KDOQI guidelines emphasize an individual approach to patients. A Life-Plan is recommended for every patient. This Life-Plan is a protocol, which takes into account the needs and preferences of every patient when choosing an approach with its timely planning, which not only emphasizes which approach to use first but also "what the next step is" when it comes to planning the first approach ${ }^{1}$. When indicating an elderly patient to an AV access, a comprehensive assessment of the patient's biological and surgical factors is necessary. In the end, the type of vascular access may be the key factor influencing survival ${ }^{6,7}$.

Previous guidelines and initiatives highlighted the fistula-first approach when choosing a vascular access for excellent patency and fewer complications in comparison with other types of vascular accesses. However, recent data questioned this due to a high degree of complications (AVF maturation failure, requiring further interventions). Therefore, this approach was re-evaluated. According to the literature, early AVF failure ranges between 20 and $60 \%$ (ref. $^{8}$ ) and made up one tenth of the AVFs in our population. A high degree of early failure may have a negative effect on an elderly patient's quality of life.

There is a higher probability that patients older than 65 will require implementation of a CVC given early failure. This is related to a higher morbidity and mortality ${ }^{9,10}$. In our population, this represented $15 \%$ of the patients, who had to be provided with a CVC after AVF failure due to an overall poor condition. The study claims that elderly patients who had the AVG created in the pre-dialysis
Table 1. Primary patency of all AV accesses.

\begin{tabular}{llrc}
\hline AV access & \multicolumn{2}{c}{ Primary patency } & $95 \% \mathrm{CI}$ \\
\hline \multirow{2}{*}{ AVF } & 12 months & $69.9 \%$ & $61.9 \%-78.0 \%$ \\
& 24 months & $62.8 \%$ & $53.8 \%-71.9 \%$ \\
\multirow{2}{*}{ AVG } & 12 months & $54.7 \%$ & $36.0 \%-73.3 \%$ \\
& 24 months & $32.2 \%$ & $10.0 \%-52.5 \%$ \\
\hline
\end{tabular}

Table 2. Primary assisted patency of all AV accesses.

\begin{tabular}{lccc}
\hline AV access & \multicolumn{2}{c}{ Primary assisted patency } & $95 \%$ CI \\
\hline AVF & 12 months & $75.4 \%$ & $68.0 \%-82.7 \%$ \\
& 24 months & $68.2 \%$ & $59.6 \%-76.8 \%$ \\
AVG & 12 months & $53.1 \%$ & $43.9 \%-71.3 \%$ \\
& 24 months & $38.5 \%$ & $18.8 \%-58.2 \%$ \\
\hline
\end{tabular}

Table 3. Secondary patency of all AV accesses.

\begin{tabular}{llrc}
\hline AV access & \multicolumn{2}{c}{ Secondary patency } & $95 \% \mathrm{CI}$ \\
\hline AVF radio-cephalic & 12 months & $70.2 \%$ & $55.1 \%-85.4 \%$ \\
& 24 months & $66.5 \%$ & $50.6 \%-82.5 \%$ \\
AVF brachio-cephalic & 12 months & $79.7 \%$ & $66.7 \%-92.6 \%$ \\
& 24 months & $65.1 \%$ & $46.7 \%-83.5 \%$ \\
AVF brachio-basilic & 12 months & $79.0 \%$ & $60.0 \%-98.0 \%$ \\
& 24 months & $69.1 \%$ & $44.6 \%-93.6 \%$ \\
AVF brachio-cephalic- & 12 months & $81.5 \%$ & $70.9 \%-92.0 \%$ \\
basilic & 24 months & $66.6 \%$ & $52.0 \%-81.3 \%$ \\
AVG & 12 months & $69.1 \%$ & $53.8 \%-84.4 \%$ \\
& 24 months & $39.7 \%$ & $20.4 \%-59.0 \%$ \\
\hline
\end{tabular}

phase were less likely to have the CVC when commencing $\mathrm{HD}$ in comparison with patients with established AVF (ref. ${ }^{6,11-13}$ ). Our results also confirm this fact.

The Drew et. al study suggests that the advantages of the fistula-first strategy significantly decreases in older patients, especially women with diabetes ${ }^{14}$. The recent cohort study, which evaluates the relation of older age, women, black ethnicity, diabetes, heart failure and a shorter onset of HS with AVF failure showed similar results ${ }^{12}$. Our results also confirm that there was a significantly shorter secondary AVF patency in women and elderly patients. Our study did not confirm a correlation with diabetes. Infection complications related to the AVG are well known; however, its correlation with age was not confirmed $^{15}$.

Lee et al. claim that elderly patients with AVG require less early intervention but more intervention in order to maintain patency after maturation in comparison with those with AVF. On the contrary, there are more early failures, frequent interventions to maintain functionality and satisfaction with a longer dependency on CVC for the AVF group. After successful use, the AVF requires less intervention and patency is somewhat longer in the first year of use in comparison with the AVG (ref. ${ }^{16}$ ). We recorded a higher number of early interventions for the AVG than the AVF. The reasons are pre-emptive percutaneous transluminal angioplasty of the AVG venous anastomosis, salvaging the vascular access and preventing its 
frequent complications - thrombosis. When evaluating secondary patency after 12 months in our population, AVG function was comparable with the AVF radio-cephalic and somewhat worse in comparison with the AVF brachiocephalic-basilic. When evaluating after 24 months, the function of the AVG was worse. This is related to the limited lifetime of a vascular prosthesis.

Properly timing the operation is essential in the older population. According to our results, more than one third of the patients were indicated for creating a vascular access later, once dialysis had occurred via CVC. Older patients usually lose renal function and there is a lower risk of progression into the final stage of kidney disease. There is also a shorter survival rate in these patients due to a higher risk of mortality ${ }^{17,18}$. In our population, patients without progression into RRT accounted for almost $2 \%$. HD was not commenced in these patients. Otherwise, when the need for HD is acute, patients would not be able to benefit from the use of early cannulated AVG, they wouldn't have to wait for AVF maturation and thereby avoid the use of the CVC (ref. ${ }^{19,20}$ ).

The advantages of the AVF over the AVG are seen when the use or excepted use of the AVF is longer than 18 months. Patients with a shorter life expectancy cannot benefit from the advantages of the AVF ( ref. $^{21}$ ).

DUS plays a key role in selecting the vascular access for HD because it provides essential information about the morphological and functional characteristics of the veins, which we want to use for creating AV accesses. Current international guidelines support the use of DUS before every AV access creation ${ }^{1}$. When measuring, it is necessary to not only focus on vein calibration but also on the quality of the arteries. According to our experience, the smaller the diameter of the artery and vein, the higher the risk of AVF early failure. Even though the artery diameters may seem sufficient, its wall may have arteriosclerotically changed and calcified, which decreases the extent of the operation's success.

Improvement in vascular access care not only in elderly patiens should be a continuous process and quality assessment could be one of the steps toward it. Generally, there is no consensus about quality assessment criteria in $\mathrm{AV}$ access procedures. In the recent article Fila B et al. tried to set a quality assessment criteria by evaluating nine worldwide guidelines by assessing surgeon experience, the patency of the AV access, the rates of AVFs in incident and prevalent HD patiens and acceptable percentage of long-term CVCs. Searched guidelines do not recommend specialty and grade of operating surgeon as well as the number of procedures per year needed for having acceptable experience and skills. The communities for vascular access were recommended to try to define realistic standards and criteria for the assessment of quality in vascular access procedures for HD at the national level in order to allow comparison of outcomes across the countries 22. Unfortunatelly it was not possible to compare our results with those presented in the article, because they are dealing with all HD patiens against our selected group of elderly HD patiens. We definately agree with the poi- gnant need of defining quality assessment criteria in this field of surgery.

The limitations of our study and the limitations of the studies stated in the literature are given by the fact that it is very difficult to lead a randomized, controlled study comparing the death rate with various access strategies (AVF, AVG). The risk of systematic errors (bias) in studies that compare clinical results according to the type of approach to HD is significant, especially if the study deals with the elderly.

\section{CONCLUSION}

The choice of AV access (AVF or AVG) should be based on the physician's best clinical judgement, taking into account the timing of the surgery, the quality of the vascular bed, co-morbidities and health conditions, so that a well-developed, useful and functional AV access is in place. For most elderly patients in the pre-dialysis phase, AVF remains their first choice. The AVG is a suitable choice for older patients, who do not have a useful vascular bed, patients who acutely require HD, or in women. It is always preferable to avoid using a CVC, which represents a high risk of infection.

Acknowledgment: Financial support: None.

Author contributions: All authors contributed equally to preparing the manuscript.

Conflict of interest statement: The authors state that there are no conflicts of interest regarding the publication of this article.

\section{REFERENCES}

1. National Kidney Foundation. KDOQI Clinical Practice Guideline for Hemodialysis Adequacy: 2015 update. Am J Kidney Dis 2015;66:884930.

2. Sidawy AN, Gray R, Besarab A, Henry M, Ascher E, Silva M Jr, Miller A, Scher L, Trerotola S, Gregory RT, Rutherford RB, Kent KC. Recommended standards for reports dealing with arteriovenous hemodialysis accesses. J Vasc Surg 2002;35:603-10.

3. Dhingra RK, Young EW, Hulbert-Shearon TE, Leavey SF, Port FK. Type of vascular access and mortality in U.S. hemodialysis patients, Kidney Int 2001;60:1443-51.

4. Pastan S, Soucle JM, McClellan WM. Vascular access and increased risc of death among hemodialysis patients, Kidney In. 2002;62:620-6.

5. Kramer A, Pippias M, Noordzij M, Stel VS, Afentakis N, Ambuhl PM, Andrusev AM, Fuster EA, Arribas Monzón FE, Åsberg A, Barbullushi M, Bonthuis M, Caskey FJ, Castro de la Nuez P, Cernevskis $\mathrm{H}$, des Grottes JM, Garneata L, Golan E, Hemmelder MH, loannou K, Jarraya F, Kolesnyk M, Komissarov K, Lassalle M, Macario F, Mahillo-Duran B, Martín de Francisco AL, Palsson R, U, Resic H, Rutkowski B, Santiuste de Pablos C, Seyahi N, Simic Ogrizovic S, Slon Roblero MF, Spustova V, Stojceva-Taneva O, Traynor J, Massy ZA, Jager KJ. The European Renal Association - European Dialysis and Transplant Association (ERA-EDTA) Registry Annual Report 2015: a summary. Clin Kidney J 2018;11:108-22.

6. Hicks CV, Canner JK, Arhuidese I, Zarkowsky DS, Qazi U, Reifsnyder T, Black JH 3rd, Malas MB. Mortality benefits of different hemodialysis access type are age dependent. J Vasc Surg 2015;61:449-56.

7. Tordoir JH, Bode AS, van Loon MM. Preferred strategy for hemodialysis access creation in elderly patients. Eur J Vasc Endovasc Surg 2015;49:738-43. 
8. Dember LM, Beck GJ, Allon M, Delmez JA, Dixon BS, Greenberg A, Himmelfarb J, Vazquez MA, Gassman JJ, Greene T, Radeva MK Braden GL, Ikizler TA, Rocco MV, Davidson IJ, Kaufman JS, Meyers CM, Kusek JW, Feldman HI. Effect of clopidogrel on early failure of arteriovenous fistulas for hemodialysis: a randomized controlled trial. JAMA 2008;299:2164-71.

9. DeSilva RN, Sandhu GS, Garg J, Goldfarb-Rumyantzev AS. Association between initial type of hemodialysis access used in the elderly and mortality. Hemodial Int 2012;16:233-41.

10. Lok CE, Allon M, Moist L, Oliver MJ, Shah H, Zimmerman D. Risk equation determining unsuccessful cannulation events and failure to maturation in arteriovenous fistulas (REDUCE FTM I). J Am Soc Nephrol 2006;17:3204-12.

11. Lee T, Thamer M, Zhang Y, Zhang Q, Allon M. Outcomes of elderly patiens after predialysis vascular access creation. J Am Soc Nephrol 2015;26:3133-40.

12. Hod T, deSilva RN, Patibandla BK, Vin Y, Brown RS, GoldfarbRumyantzev AS. Factors predicting failure of AV "fistula first" policy in the elderly. Hemodial Int 2014;18:507-15.

13. DeSilva RN, Patibandla BK, Vin Y, Narra A, Chawla V, Brown RS, Goldfarb-Rumyantzev AS. Fistula first is not always the best strategy for the elderly. J Am Soc Nephrol 2013;24:1297-304.

14. Drew DA, Lok CE, Cohen JT, Wagner M, Tangri N, Weiner DE. Vascular access choice in incident hemodialysis patients: a decision analysis. J Am Soc Nephrol 2015;26:183-91.

15. Bachleda P, Kalinova L, Utikal P, Kolar M, Hricova K, Stosova T. Infected prosthetic dialysis arteriovenous grafts: a single dialysis center study. Surg Infect 2012;13:366-70.

16. Lee T, Qian J, Thamer M, Allon M. Tradeoffs in vascular access selection in elderly patients initiating hemodialysis with a catheter. Am J Kidney Dis 2018;72:509-18.
17. Hod T, Patibandla BK, Vin Y, Brown RS, Goldfarb-Rumyantzev AS. Arteriovenous fistula placement in the elderly: when is the optimal time? J Am Soc Nephrol 2015;26:448-56.

18. Vachharajani TJ, Moossavi S, Jordan JR, Vachharajani V, Freedman BI Burkart JM. Re-evaluating the fistula first initiative in octogenarians on hemodialysis. Clin J Am Soc Nephrol 2011;6:1663-7.

19. Schmidli J, Widmer MK, Basile C, de Donato G, Gallieni M, Gibbons CP, Haage P, Hamilton G, Hedin U, Kamper L, Lazarides MK, Lindsey B, Mestres G, Pegoraro M, Roy J, Setacci C, Shemesh D, Tordoir JHM, van Loon M, Esvs Guidelines Committee, Kolh P, de Borst GJ, Chakfe N, Debus S, Hinchliffe R, Kakkos S, Koncar I, Lindholt J, Naylor R, Vega de Ceniga M, Vermassen F, Verzini F, Esvs Guidelines Reviewers, Mohaupt M, Ricco JB, Roca-Tey R. Vascular access: clinical practice guidelines of the European Society for Vascular Surgery. Eur J Vasc Endovasc Surg 2018;55:757-818.

20. Ibeas J, Roca-Tey R, Vallespin J, Moreno T, Monux G, Martí-Monros A, Del Pozo JL, Gruss E, Ramirez de Arellano M, Fontsere N, Arenas MD, Merino JL, Garcia-Revillo J, Caro P, Lopez-Espada C, Gimenez-Gaibar A, Fernandez-Lucas M, Valdes P, Fernandez-Quesada F, de la Fuente N, Hernan D, Arribas P, Sanchez de la Nieta MD, Martinez MT, Barba A. Spanish clinical guidelines on vascular access for haemodialysis. Nefrologia 2017;37(Suppl I1):1-192.

21. Lee T, Barker J, Allon M. Comparison of survival of upper arm arteriovenous fistulas and grafts after failed forarm fistula. J Am Soc Nephrol 2007;18:1936-41.

22. Fila B, Roca-Tey R, Malik J, Malovrh M, Pirozzi N, Kusztal M, Gallieni $M$, Jemcov T. Quality assessment of vascular access procedures for hemodialysis: A position paper of the Vascular Access Society based on the analysis of existing guidelines. J Vasc Access 2019;19:1-6. doi: $10.1177 / 1129729819848624$ 